\title{
A mixed analysis comparing nine minimally invasive surgeries for unresectable hepatocellular carcinoma patients
}

\author{
Ran Tao ${ }^{1}$, Xiaodan Li², Ruizhi Ran ${ }^{3}$, Zhihua Xiao ${ }^{4}$, Hongyue Zhang ${ }^{1}$, Hongyan Kong ${ }^{1}$, \\ Qiqin Song ${ }^{1}$, Yu Huang ${ }^{1}$, Likui Wang ${ }^{5}$ and Jiaquan Huang ${ }^{1}$ \\ ${ }^{1}$ Department and Institute of Infectious Disease, Tongji Hospital, Tongji Medical College, Huazhong University of Science \\ and Technology, Wuhan, Hubei, China \\ 2 Department of Infectious Diseases, The Central Hospital of Enshi Autonomous Prefecture, Enshi Clinical College of Wuhan \\ University, Wuhan, Hubei, China \\ ${ }^{3}$ Department of Interal Medicine-Oncology, The Central Hospital of Enshi Autonomous Prefecture, Enshi Clinical College of \\ Wuhan University, Wuhan, Hubei, China \\ ${ }^{4}$ Department of Medical Oncology, Hubei Cancer Hospital, Wuhan, Hubei, China \\ ${ }^{5}$ Savaid Medical School, University of Chinese Academy of Sciences Institute of Microbiology, Chinese Academy of Sciences, \\ Beijing, China \\ Correspondence to: Jiaquan Huang, email: jiaquanhuangphd@126.com \\ Keywords: unresectable hepatocellular carcinoma, transcatheter arterial chemoembolization, network meta-analysis \\ Received: June 29, $2016 \quad$ Accepted: September 21, $2016 \quad$ Published: September 29, 2016
}

\section{ABSTRACT}

Hepatocellular carcinoma (HCC) is usually managed by the transcatheter arterial chemoembolization (TACE). However, this technique has been challenged since severe complications have been observed in clinical practices. As a result, clinicians have started to seek other minimally invasive surgeries with equivalent efficacy. The corresponding surgeries were assessed by the five outcomes: complete response (CR), partial response (PR), stable disease (SD), progression disease (PD) and objective response rate (ORR). Direct meta-analysis and network meta-analysis were performed and the results were represented by odds ratios (OR), $95 \%$ confidence and credential intervals. Furthermore, the value of surface under the cumulative ranking curve (SUCRA)was calculated to provide corresponding rankings.Seventeen studies were incorporated into the network meta-analysis which indicated that TACE + externalbeam radiation therapy (EBRT) and drug-eluting beads (DEB) were better than TACE at controllingPD. TACE + EBRT demonstrated their advantages compared to TARE90Y.However, network meta-analysis comparison showed no significant difference between the corresponding eight treatments with respect to $C R, P R, S D$ and $O R R$. Moreover, the SUCRA suggested that TACE+EBRT were better than other treatments at treating unresectableHCC.Based on the present results of this network meta-analysis, TACE + EBRT was more effective than the other seven minimally invasive surgeries and therefore it is considered as the optimal treatment for HCC.

\section{INTRODUCTION}

Hepatocellular carcinoma (HCC)is one of the most lethal cancers all around the world. New medications and treatments are being developed to tackle the high morbidity and mortality of HCC [1].Currently, resection has been the first choice for managing $\mathrm{HCC}$ at early stages [2]. However, early symptoms of HCC are difficult to be identified due to the specificity of HCC. As a result, $\mathrm{HCC}$ is usually diagnosed at advanced stages in which conventional treatments such as local ablation therapy, surgical resection and liver transplantation are not feasible [3].

A previous study suggested that transcatheter arterial chemoembolization (TACE) is the first choice to treat the intermediate-stage HCC [4]. The proportion of HCC patients who achieved partial remission after TACE is approximately $62 \%$ and both tumor progression and vascular invasion were delayed significantly [5]. As suggested by various randomized controlled trials, TACE 
improved the average survival time by approximately two years compared to symptomatic treatments and systemic chemotherapies [6].Common techniques including sorafenib (SOR), percutaneous ethanol injection (PEI), high intensity focused ultrasound (HIFU), drug-eluting beads (DEB) and external-beam radiation therapy (EBRT) can be combined with other therapies. Furthermore, studies have provided evidence that introducing HIFU into TACE improved the overall survival rate compared to TACE alone [7] and the cumulative survival rate of HCC patients treated by PEI in conjunction with TACE was $12.7 \%$ higher than those treated with TACEalone [8].Some studies also suggested that introducing DEB into TACE enhanced the effectiveness of TACE and such an enhanced effectiveness is reflected by an improved survival rate as well as theinhibition of tumor progression [9].Although there was no significant difference in the total survival rate between the ethanol ablation (TEA) and TACE, it is acknowledged that TEA has more durable effects on HCC patients than TACE [10]. Recently, the yttrium-90 radio embolization (TARE-90Y)has started to replace TACEwith respect to local HCC [4].

The elegant approach of network meta-analysis enabled us to compare the efficacy of different minimally invasive surgeries by synthesizing both direct and indirect evidence obtained from randomized trials. Apart from that, the network meta-analysis provided us with a comprehensive ranking with respect to different endpoints and such a ranking may be used to distinguishing one treatment from another [11].

\section{RESULTS}

\section{Baseline characteristics of the included studies}

Initially, 1,576potentially relevant studies were identifiedfrom databases by using the predefined protocol. A total of 493studieswere excluded for duplicate publicationsor reviews, leaving 1,083 studies which passed the title and abstract review. We further excluded 671 studies since they either were not relevant to our research areas or were not qualified for the exclusion criteria. Another 395 studies were excluded as the full-text or the corresponding data were not available. Finally, 17 studies were incorporated into the network meta-analysis (Figure 1) [8-10, 12-25]. Among a total of 2,669 patients

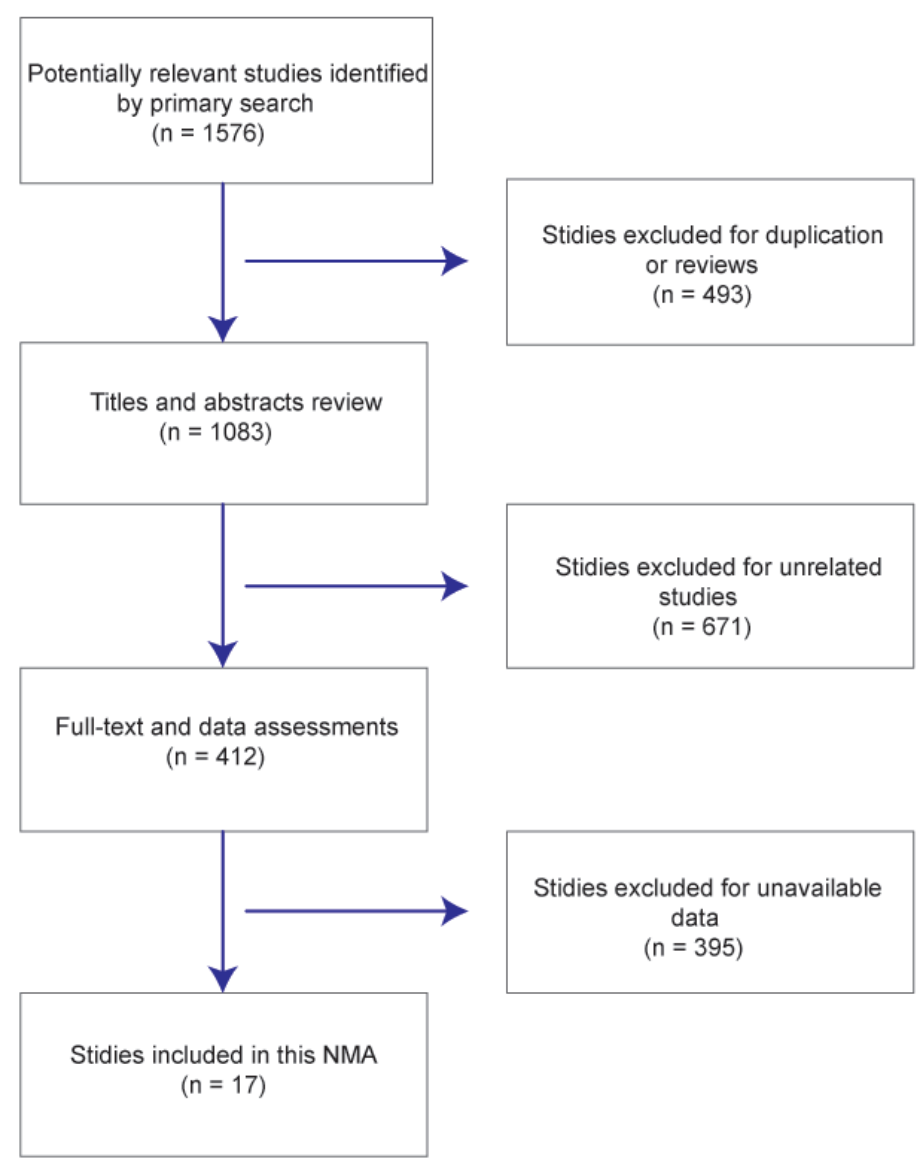

Figure 1: Literature selection flow chart. 
Table 1: Main characteristics of included studies.

\begin{tabular}{|c|c|c|c|c|c|c|c|c|c|}
\hline \multirow{2}{*}{ Study } & \multirow{2}{*}{ Region } & \multirow{2}{*}{ Year } & \multicolumn{2}{|c|}{ Participant } & \multicolumn{2}{|c|}{ Treatment } & \multicolumn{2}{|c|}{ Size } & \multirow{2}{*}{ Endpoint } \\
\hline & & & Age (SD) & Male (\%) & 1 & 2 & 1 & 2 & \\
\hline $01 \mathrm{Yu}(2014)$ & Hong Kong & 2014 & $65 \pm 20$ & 80 & TEA & TACE & 45 & 45 & (1)(2) \\
\hline 02 Wei Bai (2013) & China & 2013 & $52 \pm 12$ & 89 & $\mathrm{TACE}+\mathrm{SOR}$ & TACE & 82 & 164 & (2)(4) \\
\hline 03 Moreno-Luna (2013) & United States & 2013 & $65 \pm 15$ & 79 & TARE-90Y & TACE & 61 & 55 & (1)(3)(5) \\
\hline 04 Song (2012) & Korea & 2012 & $60 \pm 11$ & 70 & DEB-TACE & TACE & 60 & 69 & (1)(3)(5) \\
\hline 05 Song (2011) & Korea & 2011 & $64 \pm 9$ & 78 & DEB-TACE & TACE & 20 & 20 & (1)(2) \\
\hline 06 Wiggerman (2011) & Germany & 2011 & $68 \pm 9$ & 84 & DEB-TACE & TACE & 22 & 22 & (2)(4) (5) \\
\hline 07 Malagari (2011) & Greece & 2011 & $70 \pm 8$ & 77 & DEB-TACE & TACE & 41 & 43 & (2)(4)(5) \\
\hline $08 \mathrm{Li}(2010)$ & China & 2010 & $49 \pm 8$ & 80 & TACE+HIFU & TACE & 44 & 45 & (1)(3)(5) \\
\hline $09 \operatorname{Tan}(2010)$ & China & 2010 & $45 \pm 7$ & 100 & $\mathrm{TACE}+\mathrm{SOR}$ & TACE & 10 & 10 & (2)(4) (5) \\
\hline 10 Kooby $(2010)$ & United States & 2010 & $60 \pm 11$ & 83 & TARE-90Y & TACE & 27 & 44 & (2)(3)(5) \\
\hline 11 Carr (2010) & United States & 2010 & - & - & TARE-90Y & TACE & 99 & 691 & (1)(4) (4) \\
\hline 12 Lammer (2010) & Austria & 2010 & $67 \pm 9$ & 82 & DEB-TACE & TACE & 93 & 108 & (1)(3)(5) \\
\hline 13 L ewandowski & United States & 2009 & $67 \pm 7$ & 86 & TARE-90Y & TACE & 43 & 43 & (2)(4)(5) \\
\hline 14 Becker (2005) & Germany & 2005 & $64 \pm 8$ & 79 & $\mathrm{TACE}+\mathrm{PEI}$ & TACE & 27 & 25 & (2)(4) (5) \\
\hline 15 Zeng (2004) & China & 2004 & $51 \pm 12$ & 91 & $\mathrm{TACE}+\mathrm{EBRT}$ & TACE & 54 & 149 & (1)(3)(4) \\
\hline 16 Bartolozzi (1995) & Italy & 1995 & $65 \pm 6$ & 77 & $\mathrm{TACE}+\mathrm{PEI}$ & TACE & 26 & 27 & (1) (4) (5) \\
\hline
\end{tabular}

*CR: complete response; PR: partial response; SD: stable disease; PD: progression disease; ORR: objective response rate; DEB-TACE: drug-eluting beads-transcatheter arterial chemoembolization; SOR: sorafenib; TACE: transcatheter arterial chemoembolization; EBRT: external-beam radiation therapy; HIFU: high intensity focused ultrasound; PEI: percutaneous ethanol injection; TARE-90Y: yttrium-90 radioembolization; TEA: transarterial ethanol ablation.

Table 2: CR of seven minimally invasive surgeries in unresectable $\mathrm{HCC}$ according to the network meta-analysis using odds ratios (ORs) and corresponding $95 \%$ credible intervals (CrI).

\begin{tabular}{ccccccc}
\hline DEB-TACE & $0.45(0.12,1.52)$ & $4.50(0.19,190.02)$ & $1.89(0.14,24.60)$ & $2.50(0.18,31.09)$ & $2.36(0.26,19.75)$ & $0.85(0.07,10.46)$ \\
$2.24(0.66,8.08)$ & TACE & $9.94(0.58,353.40)$ & $4.27(0.44,42.24)$ & $5.55(0.56,56.70)$ & $5.28(0.93,30.63)$ & $1.90(0.24,15.87)$ \\
$0.22(0.01,5.30)$ & $0.10(0.00,1.71)$ & TACE+EBRT & $0.41(0.01,16.81)$ & $0.54(0.01,21.65)$ & $0.53(0.01,15.33)$ & $0.19(0.00,7.17)$ \\
$0.53(0.04,7.19)$ & $0.23(0.02,2.26)$ & $2.42(0.06,192.73)$ & TACE+HIFU & $1.34(0.05,35.35)$ & $1.27(0.07,22.96)$ & $0.44(0.02,9.35)$ \\
$0.40(0.03,5.46)$ & $0.18(0.02,1.78)$ & $1.84(0.05,110.87)$ & $0.75(0.03,18.41)$ & TACE+PEI & $0.94(0.06,16.57)$ & $0.34(0.02,8.28)$ \\
$0.42(0.05,3.89)$ & $0.19(0.03,1.08)$ & $1.89(0.07,89.44)$ & $0.78(0.04,14.13)$ & $1.06(0.06,17.73)$ & TARE_90Y & $0.36(0.02,5.80)$ \\
$1.17(0.10,13.69)$ & $0.53(0.06,4.13)$ & $5.26(0.14,342.35)$ & $2.26(0.11,48.82)$ & $2.95(0.12,65.87)$ & $2.78(0.17,45.38)$ & TEA \\
\hline
\end{tabular}

*DEB-TACE: drug-eluting beads-transcatheter arterial chemoembolization; TACE: transcatheter arterial chemoembolization; EBRT: external-beam radiation therapy; HIFU: high intensity focused ultrasound; PEI: percutaneous ethanol injection; TARE90Y: yttrium-90 radioembolization; TEA: transarterial ethanol ablation.

with untreated unresectable $\mathrm{HCC}, 1,560$ patients from 16 studies were treated with TACE, 256 patients from 3 studies were treated with TACE+SOR, 44 patients from 1 study were treated with TACE+HIFU, 27 patients from 1 study were treated with TACE+PEI, 236 patients from 5 studies were treated with DEB-TACE, 230 patients from4 studies were treated with TARE-90Y, 191 patients from 1 study were treated with SOR, 54 patients from 1 study were treated with TACE+EBRT, and 45 patients from 1 study were treated with TEA. The 17 correspondingstudies were published between 1995 and 2014, all of which are two-arm trials. The baseline characteristics of the incorporated studiesweresummarized in Table 1.

\section{Direct meta-analysis}

The effectiveness of the eight treatments was compared in direct meta-analysis. As compared to TACE, TACE $+\mathrm{HIFU}(\mathrm{OR}=3.84,95 \% \mathrm{CI}=1.13-13.05)$, TACE+PEI $(\mathrm{OR}=5.11,95 \% \mathrm{CI}=1.38-18.85)$ and TARE$90 \mathrm{Y}(\mathrm{OR}=4.49,95 \% \mathrm{CI}=1.33-15.09)$ were more likely to achieve aCR among patients with unresectableHCC. By contrast, DEB-TACE $(\mathrm{OR}=2.21,95 \% \mathrm{CI}=0.98-4.96)$, TACE+EBRT $(\mathrm{OR}=8.71,95 \% \mathrm{CI}=0.89-85.58)$ and TEA $(\mathrm{OR}=1.88,95 \% \mathrm{CI}=0.81-4.36)$ appeared to have similar effects with TACE on patients (Figure 2). Moreover, TACE+EBRT $(\mathrm{OR}=5.49,95 \% \mathrm{CI}=2.78-10.84)$ were more likely to induce PR compared to TACE (Figure 3). Besides that, $\mathrm{TACE}+\mathrm{SOR}(\mathrm{OR}=1.81,95 \% \mathrm{CI}=1.08-3.03)$ appeared to have stronger effects than SOR with respect to stabilizing the condition of patients (Figure 4).

When compared with TACE, ACE+SOR (OR = $0.67,95 \% \mathrm{CI}=0.40-1.13)$, $\mathrm{TACE}+\mathrm{HIFU}(\mathrm{OR}=0.45$, $95 \% \mathrm{CI}=0.14-1.44), \mathrm{TACE}+\mathrm{PEI}(\mathrm{OR}=0.81,95 \% \mathrm{CI}=$ $0.27-2.40)$ and TARE-90Y (OR $=0.93,95 \% \mathrm{CI}=0.34-$ 2.54) showed no significant difference in the probability of PD, while patients treated with DEB-TACE $(\mathrm{OR}=0.44$, $95 \% \mathrm{CI}=0.29-0.68)$ or $\mathrm{TACE}+\mathrm{EBRT}(\mathrm{OR}=0.03,95 \% \mathrm{CI}$ $=0.01-0.49$ ) were less likely to experiencePD (Figure 5). Treatments including TACE $+\mathrm{HIFU}(\mathrm{OR}=3.33,95 \% \mathrm{CI}$ $=1.37-8.09)$, DEB-TACE $(\mathrm{OR}=2.60,95 \% \mathrm{CI}=1.04-$ 6.49) and TACE+EBRT $(\mathrm{OR}=7.06,95 \% \mathrm{CI}=3.46-14.42)$ exhibited enhanced effectiveness compared to TACE with respect to ORR(Figure 6). 
Network meta-analysis

As suggested by the network meta-analysis, both TACE+EBRT and DEB-TACE $(\mathrm{OR}=0.04,95 \% \mathrm{Crl}=$ $0.01-0.52 ; \mathrm{OR}=0.33,95 \% \mathrm{Crl}=0.09-0.94)$ are more effective than TACE with respect to the inhibition of PD. Apart from that, TACE+EBRT exhibited further superiority over TARE-90Y $(\mathrm{OR}=0.04,95 \% \mathrm{Crl}=$ 0.00-0.82; Table 5). However, network meta-analysis comparison indicated no significant difference in CR, PR, SD and ORR amongtreatments (Tables 2-4,6).

Another interesting trend is that DEB-TACE and TACE+EBRTshow a higher PD ratethan TACE. Therefore, we suspected that TACE+EBRT may provide HCC patients with favorable results with respect to disease progression control whereas single TACE may not provide such effectiveness.

\section{Cumulative ranking}

Table 7 shows the relative ranking of eight minimally invasive treatments which are widely applied in clinical practices for unresectable HCC. As suggested by the corresponding SUCRA values, TACE+EBRT appeared to have the highest ranking in $\mathrm{CR}(76.50 \%)$, PR $(88.50 \%)$, PD (95.33\%) and ORR (81.13\%). TACE+SOR exhibited the highest ranking probability $(79.14 \%)$ with respect to SD. All of this evidence enabled us to conclude that TACE+EBRTmay be the most appropriate treatment for managing patients with unresectable HCC.

\section{Treatment 1 Treatment 2}

Study Events Total Events Total

OR $95 \% \mathrm{Cl}$ Weight

TACE+HIFU vs. TACE

Li (2010)

12

44

4

45

$3.84[1.13 ; 13.05]$

$9.7 \%$

TACE+PEI vs. TACE

Bartolozzi (1995)

22

26

14

27

$1:$

i

.8.

DEB-TACE vs. TACE

Song (2012)

Song (2011)

Lammer (2010)

$\begin{array}{rr}33 & 60 \\ 7 & 20 \\ 25 & 93\end{array}$

16

4

Random effects model

173

24

Heterogeneity: $I^{2}=60.9 \%$, tau ${ }^{2}=0.3017, P=0.0775$

TARE-90Y vs. TACE

Moreno-Luna (2013)
Carr (2010)

$\begin{array}{ll}7 & 61 \\ 3 & 99\end{array}$

99

Fixed effect model

160

Heterogeneity: $l^{2}=0 \%$, tau ${ }^{2}=0, P=0.5128$

TACE+EBRT vs. TACE

Zeng (2004)

3

54

1

3

TEA vs. TACE

Yu (2014)

28

45

21

149

$5.11[1.38 ; 18.85]$

$8.7 \%$

$+-$

5.11 11.38: 18.85

$8.7 \%$

$69 \quad$ i

$4.05[1.90 ; 8.62]$

$19.1 \%$

$2.15[0.52 ; 9.00]$

$1.29[0.68 ; 2.45]$

$7.5 \%$

108

197

$2.21[0.98 ; 4.96]$

$22.7 \%$

7

i

$49.3 \%$

0775 
Table 3: PR of eight minimally invasive surgeries in unresectable HCC according to the network meta-analysis using odds ratios (ORs) and corresponding $95 \%$ credible intervals (CrIs).

\begin{tabular}{|c|c|c|c|c|c|c|c|}
\hline DEB-T ACE & $0.61(0.20,1.74)$ & $3.53(0.32,34.46)$ & $0.93(0.07,10.23)$ & $0.28(0.03,2.88)$ & $1.12(0.11,8.73)$ & $0.74(0.15,3.51)$ & $0.63(0.03,10.20)$ \\
\hline $1.63(0.58,5.08)$ & $\mathrm{TACE}$ & $5.66(0.66,45.59)$ & $1.50(0.16,14.16)$ & $0.46(0.06,3.77)$ & $1.81(0.26,11.14)$ & $1.21(0.41,4.05)$ & $1.03(0.07,14.77)$ \\
\hline $0.28(0.03,3.10)$ & $0.18(0.02,1.52)$ & $\mathrm{TACE}+\mathrm{E} \mathrm{BRT}$ & $0.27(0.01,6.23)$ & $0.08(0.00,1.60)$ & $0.32(0.02,4.89)$ & $0.21(0.02,2.63)$ & $0.18(0.01,5.58)$ \\
\hline $1.07(0.10,13.39)$ & $0.67(0.07,6.10)$ & $3.76(0.16,82.86)$ & $\mathrm{TACE}+\mathrm{HIFU}$ & $0.30(0.02,6.36)$ & $1.18(0.06,19.92)$ & $0.80(0.07,10.45)$ & $0.68(0.02,19.78)$ \\
\hline $3.59(0.35,38.27)$ & $2.20(0.27,17.77)$ & $12.51(0.63,233.52)$ & $3.31(0.16,65.73)$ & $\mathrm{TACE}+\mathrm{PEI}$ & $4.07(0.21,58.33)$ & $2.68(0.26,29.01)$ & $2.25(0.07,64.38)$ \\
\hline $0.90(0.11,9.13)$ & $0.55(0.09,3.91)$ & $3.09(0.20,53.86)$ & $0.85(0.05,16.49)$ & $0.25(0.02,4.73)$ & TACE+SOR & $0.68(0.08,6.83)$ & $0.56(0.02,14.89)$ \\
\hline $1.35(0.29,6.49)$ & $0.83(0.25,2.44)$ & $4.69(0.38,48.45)$ & $1.26(0.10,15.27)$ & $0.37(0.03,3.83)$ & $1.48(0.15,12.07)$ & T ARE-90Y & $0.85(0.04,14.95)$ \\
\hline $1.60(0.10,28.84)$ & $0.97(0.07,14.08)$ & $5.56(0.18,165.28)$ & $1.47(0.05,50.56)$ & $0.44(0.02,13.84)$ & $1.77(0.07,45.74)$ & $1.18(0.07,24.57)$ & TEA \\
\hline
\end{tabular}

*DEB-TACE: drug-eluting beads-transcatheter arterial chemoembolization; SOR: sorafenib; TACE: transcatheter arterial chemoembolization; EBRT: external-beam radiation therapy; HIFU: high intensity focused ultrasound; PEI: percutaneous ethanol injection; TARE-90Y: yttrium-90 radioembolization; TEA: transarterial ethanol ablation.

Table 4: SD of eight minimally invasive surgeries in unresectable HCC according to the network meta-analysis using odds ratios (ORs) and corresponding $95 \%$ credible intervals (CrIs).

\begin{tabular}{ccccccc}
\hline DEB-TACE & $1.10(0.28,4.09)$ & $0.40(0.02,8.20)$ & $0.40(0.01,9.64)$ & $1.30(0.05,41.04)$ & $2.94(0.24,41.56)$ & $1.30(0.18,9.24)$ \\
$0.91(0.24,3.62)$ & TACE & $0.37(0.02,5.61)$ & $0.36(0.02,6.50)$ & $1.19(0.06,27.30)$ & $2.67(0.34,25.22)$ & $1.19(0.29,5.01)$ \\
$2.47(0.12,58.70)$ & $2.68(0.18,45.42)$ & TACE+EBRT & $1.00(0.02,52.42)$ & $3.25(0.05,207.23)$ & $7.06(0.25,281.84)$ & $3.21(0.15,77.92)$ \\
$2.50(0.10,67.57)$ & $2.75(0.15,51.88)$ & $1.00(0.02,57.23)$ & TACE +HIFU & $3.22(0.05,243.27)$ & $7.20(0.21,308.07)$ & $3.21(0.13,89.04)$ \\
$0.77(0.02,20.63)$ & $0.84(0.04,17.24)$ & $0.31(0.00,19.14)$ & $0.31(0.00,21.53)$ & TACE+PEI & $2.31(0.05,102.92)$ & $0.99(0.03,29.37)$ \\
$0.34(0.02,4.17)$ & $0.37(0.04,2.95)$ & $0.14(0.00,4.04)$ & $0.14(0.00,4.74)$ & $0.43(0.01,19.15)$ & TACE+SOR & $0.45(0.03,5.26)$ \\
$0.77(0.11,5.53)$ & $0.84(0.20,3.40)$ & $0.31(0.01,6.60)$ & $0.31(0.01,7.92)$ & $1.01(0.03,29.25)$ & $2.24(0.19,34.70)$ & TARE-90Y \\
\hline
\end{tabular}

*DEB-TACE: drug-eluting beads-transcatheter arterial chemoembolization; SOR: sorafenib; TACE: transcatheter arterial chemoembolization; EBRT: external-beam radiation therapy; HIFU: high intensity focused ultrasound; PEI: percutaneous ethanol injection; TARE-90Y: yttrium-90 radioembolization; TEA: transarterial ethanol ablation.

Table 5: PD of seven minimally invasive surgeries in unresectable $\mathrm{HCC}$ according to the network meta-analysis using odds ratios (ORs) and corresponding $95 \%$ credible intervals (CrIs).

\begin{tabular}{|c|c|c|c|c|c|c|}
\hline DEB-TACE & $3.04(1.07,10.57)$ & $0.11(0.01,2.44)$ & $1.28(0.11,18.66)$ & $2.19(0.26,17.71)$ & $1.63(0.22,12.32)$ & $2.83(0.56,13.98)$ \\
\hline $0.33(0.09,0.94)$ & TACE & $0.04(0.01,0.52)$ & $0.42(0.04,4.23)$ & $0.71(0.11,3.90)$ & $0.54(0.10,2.52)$ & $0.93(0.26,2.57)$ \\
\hline $9.05(0.41,405.50)$ & $27.10(1.93,1230.17)$ & TACE +EBRT & $11.94(0.32,923.49)$ & $19.67(0.65,1176.68)$ & $14.53(0.59,893.34)$ & $25.09(1.22,1280.41)$ \\
\hline $0.78(0.05,8.97)$ & $2.38(0.24,23.82)$ & $0.08(0.01,3.09)$ & TACE+HIFU & $1.68(0.09,26.23)$ & $1.28(0.07,18.61)$ & 25.12) \\
\hline $0.46(0.06,3.80)$ & $1.41(0.26,9.52)$ & $0.05(0.01,1.53)$ & $0.59(0.04,11.37)$ & TACE +PEI & $0.75(0.07,8.81)$ & $1.30(0.16,10.91)$ \\
\hline $0.61(0.08,4.49)$ & $1.85(0.40,10.42)$ & $0.07(0.01,1.69)$ & $0.78(0.05,14.23)$ & $1.33(0.11,13.70)$ & TACE +SOR & $1.75(0.23,12.39)$ \\
\hline $0.35(0.07,1.78)$ & $1.08(0.39,3.88)$ & $0.04(0.01,0.82)$ & $0.45(0.04,6.28)$ & $0.77(0.09,6.36)$ & $0.57(0.08,4.31)$ & TARE-90Y \\
\hline
\end{tabular}

*DEB-TACE: drug-eluting beads-transcatheter arterial chemoembolization; SOR: sorafenib; TACE: transcatheter arterial chemoembolization; EBRT: external-beam radiation therapy; HIFU: high intensity focused ultrasound; PEI: percutaneous ethanol injection; TARE-90Y: yttrium-90 radioembolization.

Table 6: ORR of nine minimally invasive surgeries in unresectable HCC according to the network meta-analysis using odds ratios (ORs) and corresponding $95 \%$ credible intervals (CrIs).

\begin{tabular}{|c|c|c|c|c|c|c|c|}
\hline $\mathrm{TACE}$ & $1.79(0.20,12.50)$ & $3.44(0.29,41.37)$ & $4.03(0.38,63.63)$ & $2.74(0.83,9.19)$ & $1.42(0.43,5.33)$ & $7.20(0.65,72.68)$ & $1.92(0.16,22.10)$ \\
\hline $0.56(0.08,5.09)$ & $\mathrm{TACE}+\mathrm{SOR}$ & $1.93(0.08,57.91)$ & $2.33(0.10,81.79)$ & $1.55(0.16,19.74)$ & $0.81(0.08,10.40)$ & $4.02(0.18,111.07)$ & $1.08(0.05,27.91)$ \\
\hline $0.29(0.02,3.49)$ & $0.52(0.02,11.99)$ & $\mathrm{TACE}+\mathrm{HIFU}$ & $1.20(0.04,45.77)$ & $0.79(0.05,13.19)$ & $0.42(0.03,7.44)$ & $2.08(0.06,62.72)$ & $0.57(0.02,18.80)$ \\
\hline $0.25(0.02,2.65)$ & $0.43(0.01,10.17)$ & $0.83(0.02,26.83)$ & $\mathrm{TACE}+\mathrm{PE} \mathrm{I}$ & $0.68(0.04,10.21)$ & $0.35(0.02,5.61)$ & $1.73(0.05,52.42)$ & $0.46(0.01,14.33)$ \\
\hline $0.37(0.11,1.20)$ & $0.65(0.05,6.32)$ & $1.26(0.08,20.33)$ & $1.47(0.10,28.10)$ & DEB-T ACE & $0.52(0.10,3.09)$ & $2.63(0.17,36.12)$ & $0.70(0.04,10.54)$ \\
\hline $0.70(0.19,2.33)$ & $1.23(0.10,12.32)$ & $2.41(0.13,39.14)$ & $2.88(0.18,52.65)$ & $1.91(0.32,10.32)$ & T ARE $-90 \mathrm{Y}$ & $4.99(0.30,68.79)$ & $1.35(0.08,19.55)$ \\
\hline $0.14(0.01,1.55)$ & $0.25(0.01,5.45)$ & $0.48(0.02,15.58)$ & $0.58(0.02,19.57)$ & $0.38(0.03,5.86)$ & $0.20(0.01,3.37)$ & $\mathrm{TACE}+\mathrm{EBRT}$ & $0.27(0.01,7.79)$ \\
\hline $0.52(0.05,6.37)$ & $0.92(0.04,21.01)$ & $1.76(0.05,62.91)$ & $2.19(0.07,76.26)$ & $1.42(0.09,23.59)$ & $0.74(0.05,13.32)$ & $3.71(0.13,129.28)$ & TEA \\
\hline
\end{tabular}

*DEB-TACE: drug-eluting beads-transcatheter arterial chemoembolization; SOR: sorafenib; TACE: transcatheter arterial chemoembolization; EBRT: external-beam radiation therapy; HIFU: high intensity focused ultrasound; PEI: percutaneous ethanol injection; TARE-90Y: yttrium-90 radioembolization; TEA: transarterial ethanol ablation. 
Table 7: Relative ranking of nine minimally invasive surgeries in unresectable HCC assessed by using SUCRA values.

\begin{tabular}{|c|c|c|c|c|c|}
\hline Treatment & CR & PR & SD & PD & ORR \\
\hline DEB-TACE & $38.67 \%$ & $\mathbf{6 0 . 8 8} \%$ & $46.57 \%$ & $\mathbf{6 7 . 3 3 \%}$ & $61.25 \%$ \\
\hline TACE & $8.17 \%$ & $37.50 \%$ & $50.00 \%$ & $19.50 \%$ & $21.88 \%$ \\
\hline TACE+EBRT & $\mathbf{7 6 . 5 0 \%}$ & $\mathbf{8 8 . 5 0} \%$ & $26.14 \%$ & $\mathbf{9 5 . 3 3 \%}$ & $\mathbf{8 1 . 1 3 \%}$ \\
\hline TACE+HIFU & $58.33 \%$ & $55.13 \%$ & $25.14 \%$ & $\mathbf{5 4 . 6 7 \%}$ & $\mathbf{6 3 . 7 5 \%}$ \\
\hline TACE+PEI & $\mathbf{6 6 . 0 0} \%$ & $20.63 \%$ & $55.14 \%$ & $38.00 \%$ & $\mathbf{6 8 . 5 0} \%$ \\
\hline TACE+SOR & - & $\mathbf{6 2 . 5 0} \%$ & $\mathbf{7 9 . 1 4 \%}$ & $48.00 \%$ & $46.25 \%$ \\
\hline TARE-90Y & $\mathbf{6 6 . 8 3 \%}$ & $48.25 \%$ & $\mathbf{5 6 . 8 6} \%$ & $26.33 \%$ & $38.50 \%$ \\
\hline TEA & $33.83 \%$ & $43.63 \%$ & - & - & $47.75 \%$ \\
\hline
\end{tabular}

*CR: complete response; PR: partial response; SD: stable disease; PD: progression disease; ORR: objective response rate; DEB-TACE: drug-eluting beads-transcatheter arterial chemoembolization; SOR: sorafenib; TACE: transcatheter arterial chemoembolization; EBRT: external-beam radiation therapy; HIFU: high intensity focused ultrasound; PEI: percutaneous ethanol injection; TARE-90Y: yttrium-90 radioembolization; TEA: transarterial ethanol ablation.

\section{DISCUSSION}

As one of the most common malignant tumors, the mortality ofHCC is extremely high and we have witnessed a large number of families struggling with this disease [26, 27]. In this study, we collected data from 17 studies with respect to eight minimally invasive surgical approaches in order to assess their short-term efficacy and safety. Our research objective is to rank the corresponding surgical approaches by using theelegant approach of network metaanalysis. It appears that TACE+EBRT was more effective than the other seven surgical approaches. Moreover, TACE+SOR, DEB-TACE,TACE+HIFU, TACE+PEI and TARE-90Y exhibited stronger performance than expected.

TACE has been recommended for patients who are not suitable for surgeries [28]. However, arterial occlusion in tumor tissues may trigger hypoxia and hence contribute to the over-expression of hypoxia-inducible factor 1 (HIF$1 \alpha$ ) and vascular endothelial growth factor (VEGF) [29, 30]. While the over-expression of HIF-1 $\alpha$ could cause tumor progression, invasion and metastasis [31, 32], VEGF inhibits the apoptosis of vessel endothelial cells and promotes proliferation $[33,34]$ and it triggers tumor progression by stimulating the formation of blood vessels [35]. Although our study demonstrated the short-term effects of these surgical approaches, whether these effects remain strong in the long term should be further verified $[36,37]$. As suggested by previous studies, introducing EBRT into TACE was able to enhance the effectiveness of TACE alone and hence improve the overall survival status of $\mathrm{HCC}$ patients $[23,38,39]$. Besides that, both direct and indirect evidence from the network meta-analysis reached a conclusion that TACE+EBRT were more effective than TACE alone with respect to $\mathrm{CR}, \mathrm{PR}$ and ORR. By contrast, TACE had better performance than TACE+EBRT with respect to SD and PD.On top of that, TACE+EBRT exhibited compelling results in PR, PD, CR, ORR and DCR in comparison to the other seven minimally invasive surgeries.
One major issue of TACE was contributed by the over-expression of VEGF and HIF-1 $\alpha$ which result in tumor progression, invasion and metastasis [29, $30,40]$.SOR is a multikinase inhibitor which is able to suppress tumor cell proliferation by targeting theVEGF receptor and/or the PDGF receptor [41, 42]. Our study supported the notion that introducing SOR into TACE could significantly enhance the effectiveness of TACE which was reflected by the improved PR and SD. This trend may be explained by the fact that SOR is likely to suppress the expression of VEGF and hence reduce the side effects resultedfrom TACE. Additionally, DEB-TACE also exhibited promisingresults in the SUCRA ranking. DEB-TACE is able to alleviate the local embolization resulted from HCC lesions and prolong the release of chemotherapeutic agents into adjacent tissues [43, 44]. Furthermore, DEB-TACE is able to alleviate the symptoms of HCC by releasing some antitumor lesion factors so that the side effects of TACE can be prevented [45-47]. As suggested by the corresponding ranking of SUCRA, TARE-90Y, TACE+HIFU and TACE+PEI all displayed desirable results compared to TACE alone in most of the aspects. It is known that $90 \mathrm{Y}$ is a pure $\beta$ emitter which stimulates the physical half-life of zirconium-90 to approximately 64.1 hours and such a stimulation enables it to have some excellent properties being an effective transarterial liver-directed agent [48]. More importantly, TARE-90Y is able to release radioactive particles into the liver artery without significant arterial occlusion [49] and HIFU perhaps is able to induce the complete coagulative necrosis of tumor without affecting adjacent tissues and structures [50]. On the other hand, TACE+PEI have been considered as an appropriate option for small HCC lesions provided that the diameter of tumorshould be less than $3 \mathrm{~cm}$ and such a criterion has reduced the popularity of TACE+PEI in clinical practices [51].

Nevertheless, some limitations may have uncertain impact on theoverall conclusions. For instance, four surgeries (TACE+HIFU, TACE+PEI, TACE+EBRT, 
Treatment 1 Treatment 2

TACE+SOR vs. TACE

Wei Bai (2013)

Tan (2010)

Fixed effect model

Heterogeneity: $l^{2}=46.5 \%$, $\operatorname{tau}^{2}=1.395, P=0.1717$

TACE+SOR vs. SOR

Choi (2013)

$\begin{array}{llll}17 & 164 & 8 & 191\end{array}$

TACE+HIFU vs. TACE

$\begin{array}{lllll}\text { Li (2010) } & 20 & 44 & 16 & 45\end{array}$

TACE+PEI vs. TACE

Becker (2005)

Bartolozzi (1995)

Fixed effect model

$\begin{array}{llll}1 & 27 & 1 & 25 \\ 3 & 26 & 7 & 27\end{array}$

53

52

Heterogeneity: $l^{2}=0 \%$, tau ${ }^{2}=0, P=0.5774$

DEB-TACE vs. TACE

$\begin{array}{lcccc}\text { Song }(2012) & 16 & 60 & 18 & 69 \\ \text { Song }(2011) & 10 & 20 & 2 & 20 \\ \text { Wiggerman }(2011) & 4 & 22 & 5 & 22 \\ \text { Malagari (2011) } & 2 & 41 & 1 & 43 \\ \text { Lammer }(2010) & 23 & 93 & 23 & 108 \\ \text { Fixed effect model } & & 236 & & 262\end{array}$

Heterogeneity: $l^{2}=33.9 \%$, tau ${ }^{2}=0.1665, P=0.1954$

TARE-90Y vs. TACE

$\begin{array}{lcccc}\text { Moreno-Luna (2013) } & 22 & 61 & 22 & 55 \\ \text { Kooby }(2010) & 3 & 27 & 2 & 44 \\ \text { Carr }(2010) & 38 & 99 & 380 & 691 \\ \text { Lewandowski (2009) } & 26 & 43 & 13 & 43 \\ \text { Random effects model } & & 230 & & 833 \\ \text { Heterogeneity: } l^{2}=81.7 \% \text {, tau } & 2=0.7243, P=0.0009\end{array}$

TACE+EBRT vs. TACE

Zeng (2004)

$\begin{array}{llll}38 & 54 & 45 & 149\end{array}$

TEA vs. TACE

Yu (2014)

$\begin{array}{llll}3 & 45 & 3 & 45\end{array}$

62

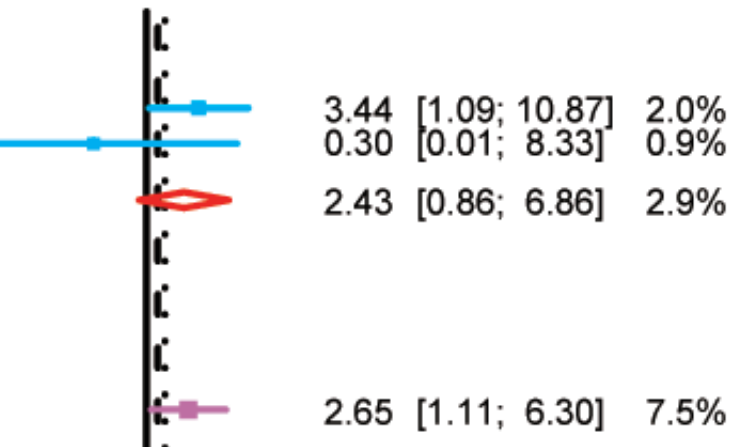

$1.51[0.64 ; 3.54] \quad 5.7 \%$

$0.92[0.05 ; 15.59] \quad 0.7 \%$

$0.37[0.08 ; 1.64] \quad 4.0 \%$

$0.45[0.12 ; 1.64] 4.7 \%$
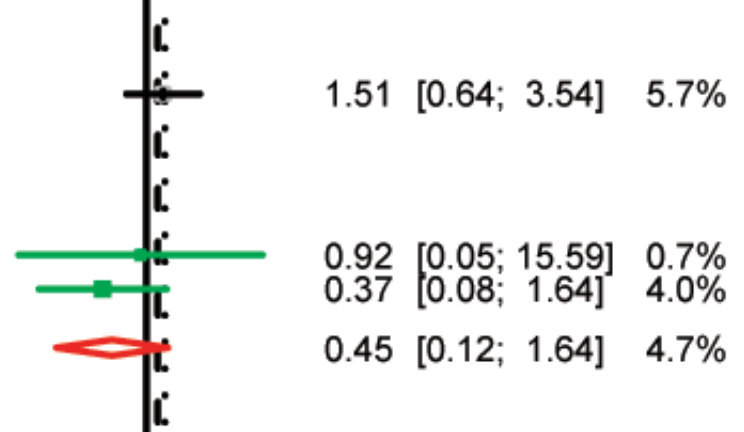
TEA) contained only one eligible trial and thus the assessment of these approaches may not be as accurate as those approaches in which a large number of trials were included. Inherent difference in studies and trials such as study design, assessment approach and surgical procedures may also have influenceon the overall results. Finally, we intentionally removed some studiesin which side effects were not mentioned and such missing studies may contain key information with respect to the efficacy and safety of these surgeries.

For summary, TACE+EBRT exhibited the most compelling results in comparison to the other seven minimally invasive surgeries. Futures studies should investigate the long-term effects of these minimally invasive surgeries on HCC patients by designingappropriate following-up studies. This study may

\section{Treatment 1 Treatment 2}

Study Events Total Events Total

OR $\quad 95 \% \mathrm{Cl}$ Weight

TACE+SOR vs. TACE

Wei Bai (2013)

Tan (2010)

Fixed effect model

Heterogeneity: $l^{2}=27.9 \%$, $\operatorname{tau}^{2}=0.1985, P=0.239$

92

$$
\begin{array}{rrrr}
40 & 82 & 60 & 164 \\
7 & 10 & 3 & 10
\end{array}
$$

174

TACE+SOR vs. SOR

Gwang Hyeon Choi (2013)95 164

$88 \quad 191$

TACE+HIFU vs. TACE

$\mathrm{Li}(2010)$

$7 \quad 44$

15

45

TACE+PEI vs. TACE

Becker (2005)

$5 \quad 27$

$4 \quad 25$

DEB-TACE vs. TACE

Song (2012)
Song (2011)
Wiggerman (2011)
Malagari (2011)
Lammer (2010)

Random effects model

Heterogeneity: $l^{2}=80.7 \%$, tau ${ }^{2}=1.548, P=0.0004$

\section{TARE-90Y vs. TACE}

Moreno-Luna (2013)
Kooby (2010)
Carr (2010)
Lewandowski (2009)

Fixed effect model

Heterogeneity: $I^{2}=0 \%$, tau ${ }^{2}=0, P=0.872$

\section{TACE+EBRT vs. TACE}

$\begin{array}{lllll}\text { Zeng (2004) } & 13 & 54 & 68 & 149\end{array}$

$\begin{array}{rrrr}22 & 61 & 16 & 55 \\ 11 & 27 & 16 & 44 \\ 35 & 99 & 199 & 691 \\ 16 & 43 & 17 & 43 \\ & 230 & & 833\end{array}$

$\begin{array}{rr}9 & 60 \\ 3 & 20 \\ 13 & 22 \\ 9 & 41 \\ 11 & 93\end{array}$
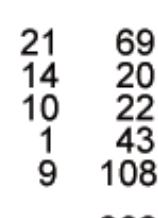

262 $\begin{array}{llll}13 & 54 & 68 & 149\end{array}$

4

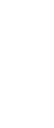

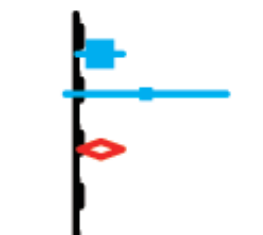

1.65

$5.44 \quad[0.80 ; 36.87] \quad 0.4 \%$

$1.81 \quad[1.08 ; 3.03] 10.7 \%$

$1.61[1.06 ; 2.45] 17.1 \%$

$0.38 \quad[0.14 ; 1.05] \quad 6.2 \%$

$1.19[0.28 ; 5.06] 1.7 \%$
$1.38 \quad[0.63 ; 3.01] \quad 5.4 \%$

$1.20 \quad 0.45 ; 3.22 \quad 3.6 \%$

$1.35 \quad 0.87 ; 2.11 \quad 16.1 \%$

0.91 [0.38; 2.16$] 5.3 \%$

$1.26[0.90 ; 1.76] 30.4 \%$
[0.17; $0.9717 .3 \%$

$0.02 ; 0.36,4.1 \%$

$0.53 ; 5.72 \quad 5.5 \%$

$[1.42 ; 98.07] 2.7 \%$

[0.26; 3.09] $26.5 \%$

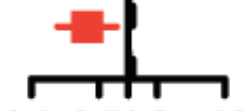

0.10 .51210

$0.38[0.19 ; 0.76] \quad 8.3 \%$

Figure 4: Forest plot of SDby using the pair-wise meta-analysis. 
provide sensible perspectives with respect to choosing the appropriate surgical approaches for individual HCC patients.

\section{MATERIALS AND METHODS}

\section{Search strategy}

Key terms such as"unresectable hepatocellular carcinoma", "transcatheter arterial chemoembolization", "sorafenib", "high intensity focused ultrasound", "percutaneous ethanol injection", "drug-eluting beads", "yttrium-90 radioembolization", "external-beam radiation therapy", "transarterial ethanol ablation" and "randomized controlled trial" (dated up to May of 2016) were used to retrieve academic articles from PubMed, Embase and Cochrane Library. No muti-arm trials were found. Additionally, a manual search of primary and secondary publication references was conducted to identify additional relevant studies.

\section{Treatment 1 Treatment 2}

Study Events Total Events Total

OR $95 \% \mathrm{Cl}$ Weight

\begin{tabular}{|c|c|c|c|c|c|c|c|}
\hline \multicolumn{8}{|c|}{ TACE+SOR vs. TACE } \\
\hline $\begin{array}{l}\text { Wei Bai (2013) } \\
\text { Tan }(2010)\end{array}$ & $\begin{array}{r}34 \\
3\end{array}$ & $\begin{array}{l}82 \\
10\end{array}$ & $\begin{array}{r}81 \\
6\end{array}$ & $\begin{array}{r}164 \\
10\end{array}$ & & $\begin{array}{l}0.73[0.42 ; 1.24] \\
0.29[0.04 ; 1.82]\end{array}$ & $\begin{array}{r}18.8 \% \\
2.5 \%\end{array}$ \\
\hline \multicolumn{4}{|c|}{$\begin{array}{lc}\text { Fixed effect model } & 92 \\
\text { Heterogeneity: } l^{2}=0 \%, \text { tau }^{2}=0, P=0.3432\end{array}$} & 174 & 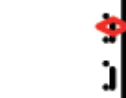 & $0.67[0.40 ; 1.13]$ & $21.3 \%$ \\
\hline \multicolumn{8}{|c|}{ TACE+HIFU vs. TACE } \\
\hline $\mathrm{Li}(2010)$ & 5 & 44 & 10 & 45 & & $0.45[0.14 ; 1.44]$ & $5.2 \%$ \\
\hline \multicolumn{8}{|l|}{ TACE+PEI vs. TACE } \\
\hline $\begin{array}{l}\text { Becker (2005) } \\
\text { Bartolozzi (1995) }\end{array}$ & $\begin{array}{r}20 \\
0\end{array}$ & $\begin{array}{l}27 \\
26\end{array}$ & $\begin{array}{r}18 \\
2\end{array}$ & $\begin{array}{l}25 \\
27\end{array}$ & & $\begin{array}{l}1.11[0.33 ; 3.79] \\
0.19[0.01 ; 4.21]\end{array}$ & $\begin{array}{l}2.9 \% \\
1.4 \%\end{array}$ \\
\hline \multicolumn{8}{|c|}{ Heterogeneity: $P^{2}=8.4 \%$, tau $^{2}=0.1312, P=0.2961$} \\
\hline \multicolumn{8}{|c|}{ DEB-TACE vs. TACE } \\
\hline $\begin{array}{l}\text { Song }(2012) \\
\text { Wiggerman }(2011) \\
\text { Malagari }(2011) \\
\text { Lammer }(2010)\end{array}$ & $\begin{array}{r}2 \\
2 \\
17 \\
30\end{array}$ & $\begin{array}{l}60 \\
22 \\
41 \\
93\end{array}$ & $\begin{array}{r}14 \\
7 \\
29 \\
44\end{array}$ & $\begin{array}{r}69 \\
22 \\
43 \\
108\end{array}$ & & $\begin{array}{l}0.14[0.03 ; 0.62] \\
0.21[0.04 ; 1.18] \\
0.34[0.14 ; 0.83] \\
0.69[0.39 ; 1.24]\end{array}$ & $\begin{array}{r}7.5 \% \\
3.8 \% \\
9.9 \% \\
16.4 \%\end{array}$ \\
\hline Fixed effect model & & 216 & & 242 & d & $0.44[0.29 ; 0.68]$ & $37.6 \%$ \\
\hline \multicolumn{8}{|c|}{ Heterogeneity: $P^{2}=46.6 \%$, tau $^{2}=0.2385, P=0.1315$} \\
\hline \multicolumn{8}{|l|}{ TARE-90Y vs. TACE } \\
\hline $\begin{array}{l}\text { Moreno-Luna (2013) } \\
\text { Kooby (2010) } \\
\text { Carr (2010) } \\
\text { Lewandowski (2009) }\end{array}$ & $\begin{array}{r}5 \\
9 \\
23 \\
1\end{array}$ & $\begin{array}{r}61 \\
27 \\
99 \\
43 \\
230\end{array}$ & $\begin{array}{r}7 \\
16 \\
75 \\
5\end{array}$ & $\begin{array}{r}55 \\
44 \\
691 \\
43\end{array}$ & & $\begin{array}{l}0.61[0.18 ; 2.05] \\
0.88[0.32 ; 2.40] \\
2.49[1.47 ; 4.20] \\
0.18[0.02 ; 1.62] \\
0.93[0.34 ; 254]\end{array}$ & $\begin{array}{r}7.6 \% \\
8.7 \% \\
11.4 \% \\
3.9 \% \\
31.5 \%\end{array}$ \\
\hline \multirow{2}{*}{\multicolumn{6}{|c|}{$\begin{array}{l}\text { Random effects model } \\
\text { Heterogeneity: } l^{2}=71.6 \%, \operatorname{tau}^{2}=0.693, P=0.0143\end{array}$}} & & \\
\hline TACE+EBRT vs. T/ & & & & & & & \\
\hline Zeng (2004) & 0 & 54 & 35 & $149-$ & إل & $0.03[0.00 ; 0.49]$ & $11.2 \%$ \\
\hline
\end{tabular}

Figure 5: Forest plot of PDby using the pair-wise meta-analysis. 


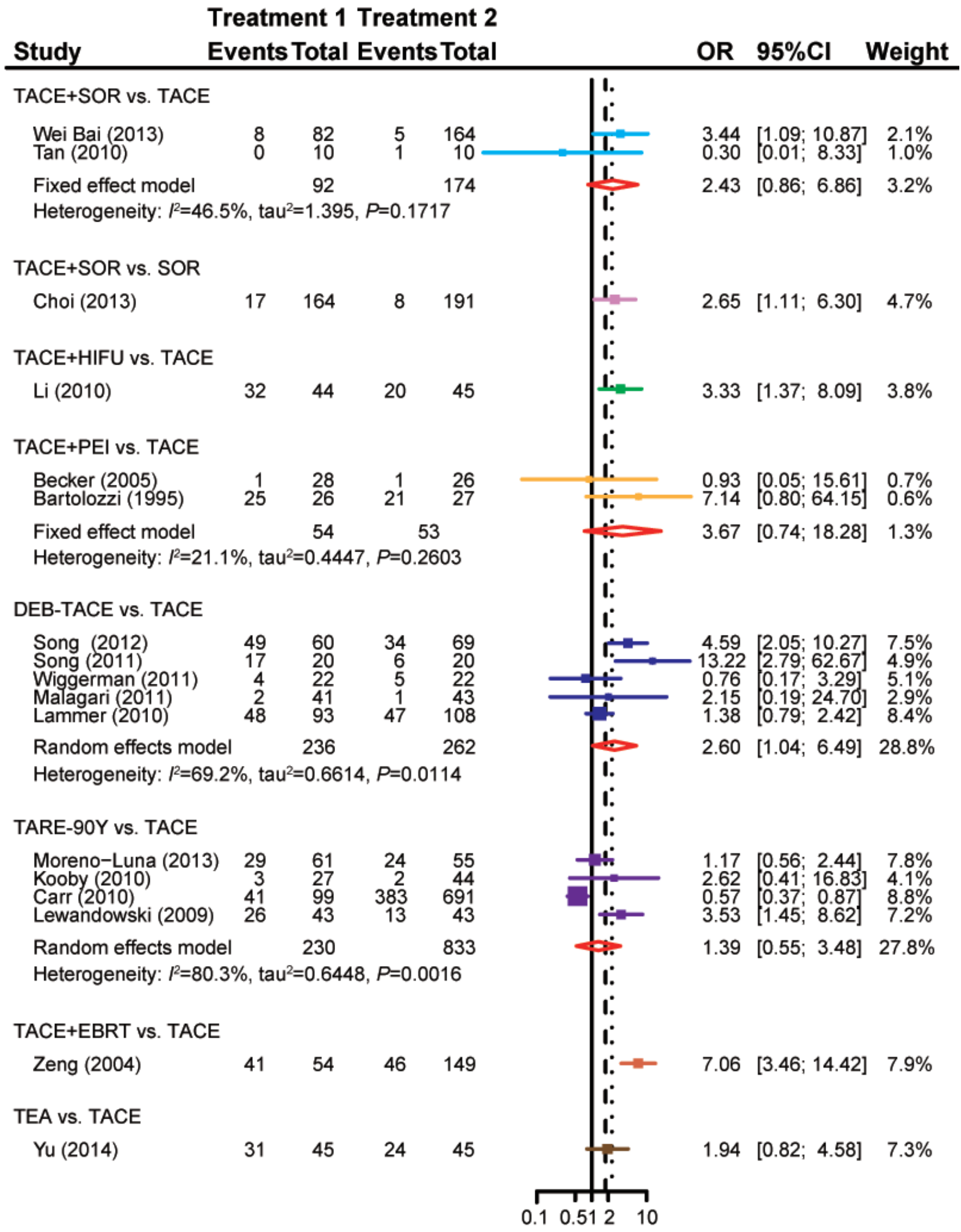

Figure 6: Forest plot of ORRby using thepair-wise meta-analysis. 


\section{Inclusion and exclusion criteria}

In the meta-analysis, studies that met the following criteria were included: (i) randomized controlled trials; (ii) one or more of the following interventions were involved in the trials: TACE, TACE+SOR, SOR, TACE+HIFU, TACE+PEI, DEB-TACE, TARE-90Y, TACE+EBRT and TEA; (iii) patients involved were over 18yearsold with unresectableHCC; (iv) no operative interventions had been conducted before the trials were conducted; (v) one or more of the following outcomes were assessed: complete response (CR), partial response (PR), stable disease (SD), progression disease $(\mathrm{PD})$ and objective response rate (ORR).

However, studies were excluded if any of the following conditions were satisfied: (i) patients involved failed to perform normal activities or did not have normal functioning kidneys, lungs or heart; (ii) treatments for unresactableHCC, such as surgery, medication, radiotherapy and others, had been given; (iii) unqualified literatures which did not have academic integrity (e.g. incorrectly matched case-control studies).

\section{Data extraction and outcome measures}

The corresponding data was extracted by two reviewers independently, and then were entered into Excel spreadsheets that were created by the third reviewer. The data were extracted including the author, region, year, treatment, size of sample and endpoints. Disagreements between the two reviewers, if any, were resolved by consensus or with the help of the third reviewer.

Main outcome measures include: (i) CR: all of clinically visible pathological features were eliminated for more than 4weeks and tumor marker was monitored without new pathological changes; (ii) PR: sum of the maximum diameter of tumor clinical lesions had decreased by more than $30 \%$ or the product of the maximum diameter and maximum vertical diameter had decreased by more than $50 \%$, and had lasted for more than 4 weeks; (iii) PD: sum of the maximum diameter of tumor clinical lesions had increased by more than $20 \%$, the product of two maximum diameters had increased by $25 \%$, or new lesions had developed; (iv) SD: reduced sum or product of the two maximum diameters were below the standard of PR, or the increased amount was below the standard of $\mathrm{PD}$; (v) $\mathrm{ORR}=\mathrm{CR}+\mathrm{PR}$ : the sum of complete response and partial response cases.

\section{Statistical analysis}

Initially, we performed direct meta-analysis with $\mathrm{R}$ software (V3.2.1). Binary outcomes were then compared in terms of odds ratios (OR) and $95 \%$ confidence interval
(CI). Inter-study heterogeneity was identified using the $\mathrm{Q}$ test and $I^{2}$ test. If there was no significant heterogeneity among studies, a fixed-effects model was selected for analysis; otherwise a random-effects model was selected for analysis [52]. Next, network meta-analysis was performed by using the GEMTC (V0.6) package and the corresponding results were combined with those obtained from the DerSimonian-Laird random-effects model and Monte Carlo Markov Chain (MCMC). We used R software (V3.2.1) in order to produce the surface under the cumulative ranking curve (SUCRA) and calculated the ranking of different interventions [53]. For each intervention, efficacy and safety outcomes were ranked by the SUCRA: a higher value of SUCRA indicated a higher ranking. All the calculations were conducted by using $\mathrm{R}$ software (V3.2.1), GEMTC package (V0.6) and Open BUGS (V3.4.0).

\section{ACKNOWLEDGMENTS}

It is funded by the National Science and Technology Major Project (No. 2014ZX10005001).

\section{CONFLICTS OF INTEREST}

The authors declare no conflict of interest.

\section{REFERENCES}

1. Chang TS, Chen CL, Wu YC, Liu JJ, Kuo YC, Lee KF, Lin SY, Lin SE, Tung SY, Kuo LM, Tsai YH and Huang YH. Inflammation Promotes Expression of Stemness-Related Properties in HBV-Related Hepatocellular Carcinoma. PloS one. 2016; 11(2):e0149897.

2. Yamakado K, Nakatsuka A, Akeboshi M, Shiraki $\mathrm{K}$, Nakano $\mathrm{T}$ and Takeda K. Combination therapy with radiofrequency ablation and transcatheter chemoembolization for the treatment of hepatocellular carcinoma: Short-term recurrences and survival. Oncology reports. 2004; 11(1):105-109.

3. Lin S, Hoffmann $\mathrm{K}$ and Schemmer P. Treatment of hepatocellular carcinoma: a systematic review. Liver cancer. 2012; 1(3-4):144-158.

4. Moreno-Luna LE, Yang JD, Sanchez W, Paz-Fumagalli R, Harnois DM, Mettler TA, Gansen DN, de Groen PC, Lazaridis KN, Narayanan Menon KV, Larusso NF, Alberts SR, Gores GJ, et al. Efficacy and safety of transarterial radioembolization versus chemoembolization in patients with hepatocellular carcinoma. Cardiovascular and interventional radiology. 2013; 36(3):714-723.

5. Lammer J, Malagari K, Vogl T, Pilleul F, Denys A, Watkinson A, Pitton M, Sergent G, Pfammatter T, Terraz S, Benhamou Y, Avajon Y, Gruenberger T, et al. Prospective randomized study of doxorubicin-eluting-bead embolization 
in the treatment of hepatocellular carcinoma: results of the PRECISION V study. Cardiovascular and interventional radiology. 2010; 33(1):41-52.

6. Llovet JM and Bruix J. Systematic review of randomized trials for unresectable hepatocellular carcinoma: Chemoembolization improves survival. Hepatology. 2003; 37(2):429-442.

7. Li C, Zhang W, Zhang R, Zhang L, Wu P and Zhang F. Therapeutic effects and prognostic factors in high-intensity focused ultrasound combined with chemoembolisation for larger hepatocellular carcinoma. European journal of cancer. 2010; 46(13):2513-2521.

8. Becker G, Soezgen T, Olschewski M, Laubenberger J, Blum HE and Allgaier HP. Combined TACE and PEI for palliative treatment of unresectable hepatocellular carcinoma. World journal of gastroenterology. 2005; 11(39):6104-6109.

9. Song MJ, Chun HJ, Song do S, Kim HY, Yoo SH, Park $\mathrm{CH}$, Bae SH, Choi JY, Chang UI, Yang JM, Lee HG and Yoon SK. Comparative study between doxorubicin-eluting beads and conventional transarterial chemoembolization for treatment of hepatocellular carcinoma. Journal of hepatology. 2012; 57(6):1244-1250.

10. Yu SC, Hui JW, Hui EP, Chan SL, Lee KF, Mo F, Wong J, Ma B, Lai P, Mok T and Yeo W. Unresectable hepatocellular carcinoma: randomized controlled trial of transarterial ethanol ablation versus transcatheter arterial chemoembolization. Radiology. 2014; 270(2):607-620.

11. Zhu GQ, Shi KQ, Yu HJ, He SY, Braddock M, Zhou MT, Chen YP and Zheng MH. Optimal adjuvant therapy for resected hepatocellular carcinoma: a systematic review with network meta-analysis. Oncotarget. 2015; 6:18151-18161. doi: 10.18632/oncotarget.4098.

12. Choi GH, Shim JH, Kim MJ, Ryu MH, Ryoo BY, Kang YK, Shin YM, Kim KM, Lim YS and Lee HC. Sorafenib alone versus sorafenib combined with transarterial chemoembolization for advanced-stage hepatocellular carcinoma: results of propensity score analyses. Radiology. 2013; 269(2):603-611.

13. Moreno-Luna LE, Yang JD, Sanchez W, Paz-Fumagalli R, Harnois DM, Mettler TA, Gansen DN, De Groen PC, Lazaridis KN, Narayanan Menon KV, Larusso NF, Alberts SR, Gores GJ, et al. Efficacy and safety of transarterial radioembolization versus chemoembolization in patients with hepatocellular carcinoma. Cardiovascular and interventional radiology. 2013; 36(3):714-723.

14. Song MJ, Park CH, Kim JD, Kim HY, Bae SH, Choi JY, Yoon SK, Chun HJ, Choi BG and Lee HG. (2011). Drugeluting bead loaded with doxorubicin versus conventional Lipiodol-based transarterial chemoembolization in the treatment of hepatocellular carcinoma: a case-control study of Asian patients. Eur J Gastroenterol Hepatol, pp. 521-527.

15. Wiggermann P, Sieron D, Brosche C, Brauer T, Scheer F, Platzek I, Wawrzynek W and Stroszczynski C. Transarterial Chemoembolization of Child-A hepatocellular carcinoma: drug-eluting bead TACE (DEB TACE) vs. TACE with cisplatin/lipiodol (cTACE). Medical science monitor. 2011; 17(4):CR189-195.

16. Malagari K, Pomoni M, Kelekis A, Pomoni A, Dourakis S, Spyridopoulos T, Moschouris H, Emmanouil E, Rizos $\mathrm{S}$ and Kelekis D. Prospective randomized comparison of chemoembolization with doxorubicin-eluting beads and bland embolization with BeadBlock for hepatocellular carcinoma. Cardiovascular and interventional radiology. 2010; 33(3):541-551.

17. Li C, Zhang $\mathrm{W}$, Zhang R, Zhang L, Wu P and Zhang F. Therapeutic effects and prognostic factors in high-intensity focused ultrasound combined with chemoembolisation for larger hepatocellular carcinoma. European journal of cancer. 2010; 46(13):2513-2521.

18. Tan WF, Qiu ZQ, Yu Y, Ran RZ, Yi B, Lau WY, Liu C, Qiu YH, Feng FL, Wang JH, Yan PN, Zhang BH, Wu MC, et al. Sorafenib extends the survival time of patients with multiple recurrences of hepatocellular carcinoma after liver transplantation. Acta pharmacologica Sinica. 2010; 31(12):1643-1648.

19. Kooby DA, Egnatashvili V, Srinivasan S, Chamsuddin A, Delman KA, Kauh J, Staley CA, 3rd and Kim HS. Comparison of yttrium-90 radioembolization and transcatheter arterial chemoembolization for the treatment of unresectable hepatocellular carcinoma. Journal of vascular and interventional radiology : JVIR. 2010; 21(2):224-230.

20. Carr BI, Kondragunta V, Buch SC and Branch RA. Therapeutic equivalence in survival for hepatic arterial chemoembolization and yttrium 90 microsphere treatments in unresectable hepatocellular carcinoma: a two-cohort study. Cancer. 2010; 116(5):1305-1314.

21. Lammer J, Malagari K, Vogl T, Pilleul F, Denys A, Watkinson A, Pitton M, Sergent G, Pfammatter T, Terraz S, Benhamou Y, Avajon Y, Gruenberger T, et al. Prospective randomized study of doxorubicin-eluting-bead embolization in the treatment of hepatocellular carcinoma: results of the PRECISION V study. Cardiovascular and interventional radiology. 2010; 33(1):41-52.

22. Lewandowski RJ, Kulik LM, Riaz A, Senthilnathan S, Mulcahy MF, Ryu RK, Ibrahim SM, Sato KT, Baker T, Miller FH, Omary R, Abecassis M and Salem R. A comparative analysis of transarterial downstaging for hepatocellular carcinoma: chemoembolization versus radioembolization. American journal of transplantation. 2009; 9(8):1920-1928.

23. Zeng ZC, Tang ZY, Fan J, Zhou J, Qin LX, Ye SL, Sun HC, Wang BL, Yu Y, Wang JH and Guo W. A comparison of chemoembolization combination with and without radiotherapy for unresectable hepatocellular carcinoma. Cancer J. 2004; 10(5):307-316.

24. Bartolozzi C, Lencioni R, Caramella D, Vignali C, Cioni R, Mazzeo S, Carrai M, Maltinti G, Capria A and Conte PF. Treatment of large HCC: transcatheter 
arterial chemoembolization combined with percutaneous ethanol injection versus repeated transcatheter arterial chemoembolization. Radiology. 1995; 197(3):812-818.

25. Bai W, Wang YJ, Zhao Y, Qi XS, Yin ZX, He CY, Li RJ, Wu KC, Xia JL, Fan DM and Han GH. Sorafenib in combination with transarterial chemoembolization improves the survival of patients with unresectable hepatocellular carcinoma: a propensity score matching study. Journal of digestive diseases. 2013; 14(4):181-190.

26. Venook AP, Papandreou C, Furuse J and de Guevara LL. The incidence and epidemiology of hepatocellular carcinoma: a global and regional perspective. The oncologist. 2010; 15 Suppl 4:5-13.

27. Ferenci P, Fried M, Labrecque D, Bruix J, Sherman M, Omata M, Heathcote J, Piratsivuth T, Kew M, Otegbayo JA, Zheng SS, Sarin S, Hamid S, et al. World Gastroenterology Organisation Guideline. Hepatocellular carcinoma (HCC): a global perspective. Journal of gastrointestinal and liver diseases. 2010; 19(3):311-317.

28. Bruix J, Sala M and Llovet JM. Chemoembolization for hepatocellular carcinoma. Gastroenterology. 2004; 127(5 Suppl 1):S179-188.

29. Chen C, Wang J, Liu R and Qian S. RNA interference of hypoxia-inducible factor-1 alpha improves the effects of transcatheter arterial embolization in rat liver tumors. Tumour biology. 2012; 33(4):1095-1103.

30. Jia ZZ, Jiang GM and Feng YL. Serum HIF-1alpha and VEGF levels pre- and post-TACE in patients with primary liver cancer. Chinese medical sciences journal. 2011; 26(3):158-162.

31. Matsuo Y, Ding Q, Desaki R, Maemura K, Mataki Y, Shinchi H, Natsugoe S and Takao S. Hypoxia inducible factor-1 alpha plays a pivotal role in hepatic metastasis of pancreatic cancer: an immunohistochemical study. Journal of hepato-biliary-pancreatic sciences. 2014; 21(2):105-112.

32. Koperek O, Akin E, Asari R, Niederle B and Neuhold N. Expression of hypoxia-inducible factor 1 alpha in papillary thyroid carcinoma is associated with desmoplastic stromal reaction and lymph node metastasis. Virchows Archiv. 2013; 463(6):795-802.

33. Winiarski BK, Wolanska KI, Rai S, Ahmed T, Acheson $\mathrm{N}$, Gutowski NJ and Whatmore JL. Epithelial ovarian cancer-induced angiogenic phenotype of human omental microvascular endothelial cells may occur independently of VEGF signaling. Translational oncology. 2013; 6(6):703714.

34. Li J, Xu Y, Long XD, Wang W, Jiao HK, Mei Z, Yin QQ, Ma LN, Zhou AW, Wang LS, Yao M, Xia Q and Chen GQ. Cbx4 governs HIF-1alpha to potentiate angiogenesis of hepatocellular carcinoma by its SUMO E3 ligase activity. Cancer cell. 2014; 25(1):118-131.

35. Srabovic N, Mujagic Z, Mujanovic-Mustedanagic J, Softic A, Muminovic Z, Rifatbegovic A and Begic L. Vascular endothelial growth factor receptor-1 expression in breast cancer and its correlation to vascular endothelial growth factor a. International journal of breast cancer. 2013; 2013:746749.

36. Chang HC, Lin YM, Yen AM, Chen SL, Wu WY, Chiu SY, Fann JC, Lin YS, Chen HH and Liao CS. Predictors of long-term survival in hepatocellular carcinomas: A longitudinal follow-up of 108 patients with small tumors. Anticancer research. 2013; 33(11):5171-5178.

37. Mazioti A, Gatselis NK, Rountas C, Zachou K, Filippiadis DK, Tepetes K, Koukoulis GK, Fezoulidis I and Dalekos GN. Safety and efficacy of transcatheter arterial chemoemboliazation in the real-life management of unresectable hepatocellular carcinoma. Hepatitis monthly. 2013; 13(8):e7070.

38. Zeng ZC, Fan J, Tang ZY, Zhou J, Qin LX, Wang JH, Sun HC, Wang BL, Zhang JY, Jiang GL and Wang YQ. A comparison of treatment combinations with and without radiotherapy for hepatocellular carcinoma with portal vein and/or inferior vena cava tumor thrombus. International journal of radiation oncology, biology, physics. 2005; 61(2):432-443.

39. Zeng ZC, Tang ZY, Yang BH, Fan J, Qin LX, Jiang GL, Zhou J, Sun HC, Cheng JM and Wang JH. Radiation therapy for the locoregional lymph node metastases from hepatocellular carcinoma, phase I clinical trial. Hepatogastroenterology. 2004; 51(55):201-207.

40. Sergio A, Cristofori C, Cardin R, Pivetta G, Ragazzi R, Baldan A, Girardi L, Cillo U, Burra P, Giacomin A and Farinati F. Transcatheter arterial chemoembolization (TACE) in hepatocellular carcinoma (HCC): the role of angiogenesis and invasiveness. The American journal of gastroenterology. 2008; 103(4):914-921.

41. Chang YS, Adnane J, Trail PA, Levy J, Henderson A, Xue D, Bortolon E, Ichetovkin M, Chen C, McNabola A, Wilkie D, Carter CA, Taylor IC, et al. Sorafenib (BAY 43-9006) inhibits tumor growth and vascularization and induces tumor apoptosis and hypoxia in RCC xenograft models. Cancer chemotherapy and pharmacology. 2007; 59(5):561574.

42. Copur MS. Sorafenib in advanced hepatocellular carcinoma. The New England journal of medicine. 2008; 359(23):2498; author reply 2498-2499.

43. Lewis AL, Gonzalez MV, Lloyd AW, Hall B, Tang Y, Willis SL, Leppard SW, Wolfenden LC, Palmer RR and Stratford PW. DC bead: in vitro characterization of a drugdelivery device for transarterial chemoembolization. Journal of vascular and interventional radiology. 2006; 17(2 Pt 1):335-342.

44. Lewis AL, Taylor RR, Hall B, Gonzalez MV, Willis SL and Stratford PW. Pharmacokinetic and safety study of doxorubicin-eluting beads in a porcine model of hepatic arterial embolization. Journal of vascular and interventional radiology. 2006; 17(8):1335-1343. 
45. Jordan O, Denys A, De Baere T, Boulens N and Doelker E. Comparative study of chemoembolization loadable beads: in vitro drug release and physical properties of DC bead and hepasphere loaded with doxorubicin and irinotecan. Journal of vascular and interventional radiology. 2010; 21(7):10841090.

46. Gupta S, Wright KC, Ensor J, Van Pelt CS, Dixon KA and Kundra V. Hepatic arterial embolization with doxorubicinloaded superabsorbent polymer microspheres in a rabbit liver tumor model. Cardiovascular and interventional radiology. 2011; 34(5):1021-1030.

47. Liu DM, Kos S, Buczkowski A, Kee S, Munk PL, Klass D and Wasan E. Optimization of doxorubicin loading for superabsorbent polymer microspheres: in vitro analysis. Cardiovascular and interventional radiology. 2012; 35(2):391-398.

48. Sato K, Lewandowski RJ, Bui JT, Omary R, Hunter RD, Kulik L, Mulcahy M, Liu D, Chrisman H, Resnick S, Nemcek AA, Jr., Vogelzang $\mathrm{R}$ and Salem R. Treatment of unresectable primary and metastatic liver cancer with yttrium-90 microspheres (TheraSphere): assessment of hepatic arterial embolization. Cardiovascular and interventional radiology. 2006; 29(4):522-529.
49. Campbell AM, Bailey IH and Burton MA. Analysis of the distribution of intra-arterial microspheres in human liver following hepatic yttrium-90 microsphere therapy. Physics in medicine and biology. 2000; 45(4):1023-1033.

50. Zhang L, Zhu H, Jin C, Zhou K, Li K, Su H, Chen W, Bai $\mathrm{J}$ and Wang Z. High-intensity focused ultrasound (HIFU): effective and safe therapy for hepatocellular carcinoma adjacent to major hepatic veins. European radiology. 2009; 19(2):437-445.

51. Tesdal IK, Wikstrom M, Flechtenmacher C, Filser $\mathrm{T}$ and Dueber C. Percutaneous treatment of hepatocellular carcinoma in patients with transjugular intrahepatic portosystemic shunts. Cardiovascular and interventional radiology. 2006; 29(5):778-784.

52. Higgins JP and Thompson SG. Quantifying heterogeneity in a meta-analysis. Statistics in medicine. 2002; 21(11):15391558 .

53. Donegan S, Williamson $\mathrm{P}, \mathrm{D}$ 'Alessandro $\mathrm{U}$ and Tudur Smith C. Assessing key assumptions of network metaanalysis: a review of methods. Research synthesis methods. 2013; 4(4):291-323. 